\title{
Incomplete myocardial rupture following inferolateral myocardial infarction
}

\author{
Maciej Kabaj ${ }^{1}$, Mikołaj Berezowski ${ }^{2}$, Roman Przybylski ${ }^{3}$, Agnieszka Witkowska ${ }^{4}$, Tomasz Brzostowicz ${ }^{5}$, \\ Wojciech Kosmala ${ }^{1}$
}

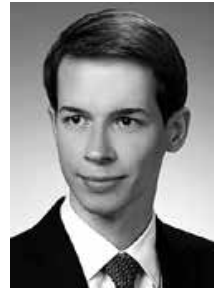

\author{
1Department of Cardiology, Wroclaw Medical University, Wroclaw, Poland \\ 2Department of Cardiac Surgery, Wroclaw Medical University, Wroclaw, Poland \\ ${ }^{3}$ Department of Heart Transplantation and Mechanical Circulatory Support, Wroclaw Medical University, Wroclaw, Poland \\ ${ }^{4}$ Centre for Heart Diseases, University Hospital, Wroclaw, Poland \\ ${ }^{5}$ RADOMED - CMR Unit, Nysa, Poland
}

Kardiochir Torakochir Pol 2020; 17 (2): 105-106

A 35-year-old man with no significant past medical history was admitted to the cardiology department with inferolateral ST-elevation myocardial infarction. Coronary angiogram performed immediately after admission (approximately 11-12 hours after the onset of chest pain) revealed right coronary artery occlusion, which was successfully revascularized by percutaneous coronary intervention $(\mathrm{PCl})$ with drug-eluting stent implantation. Postprocedural transthoracic echocardiography showed left ventricular (LV) function abnormalities with ejection fraction reduced to $38 \%$, akinesis of inferior, inferolateral and lateral segments, as well as an aneurysmatic bulge in the inferolateral wall, with wall thickness decreased to $3-4 \mathrm{~mm}$. No pericardial effusion or evidence of cardiac tamponade were found. In view of these findings, urgent cardiac magnetic resonance (CMR) imaging was performed to further explore the morphology of LV wall thinning. This examination revealed incomplete free wall rupture (FWR) encompassing mid inferior, inferolateral and lateral segments (Figures 1 A, B).

The patient was immediately transferred to the cardiac surgery department and operating room. After median sternotomy and careful opening of the pericardium, cardiopulmonary bypass was instituted. Subsequently, cardioplegic arrest was achieved and the incomplete FWR of the inferior wall was visualized. The defect was approximately $5 \times 2 \mathrm{~cm}$, covered by a thin layer of myocardium (Figure $2 \mathrm{~A}$ ). In the absence of active bleeding, it was decided to use a pericardial patch (Bio Integral $8 \times 12 \mathrm{~cm}$ ) to cover the area of myocardial tear and muscle necrosis. Its borders were placed on healthy myocardium in the peri-necrotic area and fixed onto the myocardial surface with surgical glue (BioGlue, Cryolife) (Figure 2 B). There were no complications during the operative and postoperative period. On the $2^{\text {nd }}$ day after the operation, the patient was moved from the intensive care unit to the regular ward, and on the $14^{\text {th }}$ day, discharged from the hospital.
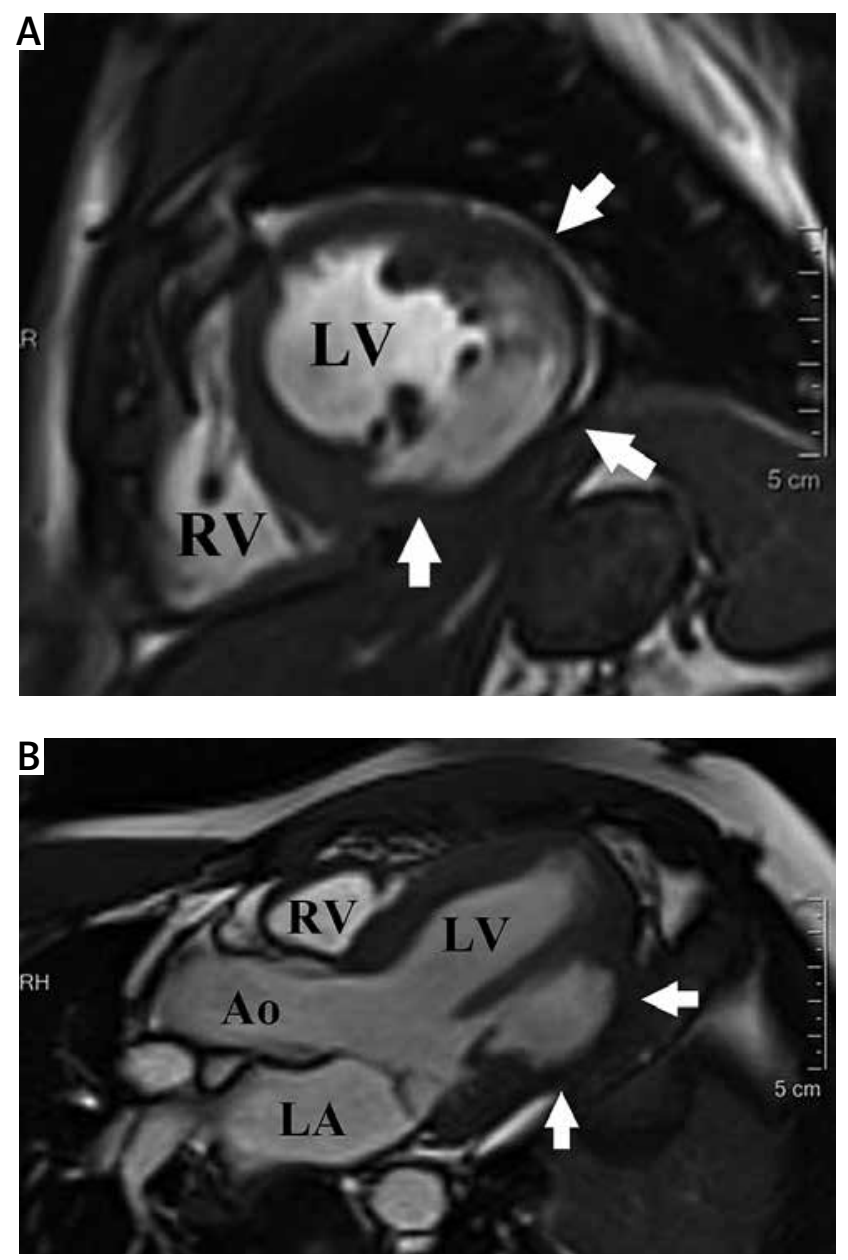

Figure 1. Cardiac magnetic resonance: short-axis view at the papillary muscle level (A) and 3-chamber view (B) demonstrating an incomplete wall rupture encompassing mid inferior, inferolateral and lateral segments of the left ventricle (arrows)

Ao - aorta, LA - left atrium, LV - left ventricle, RV - right ventricle.

Address for correspondence: Wojciech Kosmala MD, PhD, Department of Cardiology, Wroclaw Medical University, Wroclaw, Poland, e-mail: wojciech.kosmala@umed.wroc.pl

Received: 19.03.2020, accepted: 20.04.2020. 

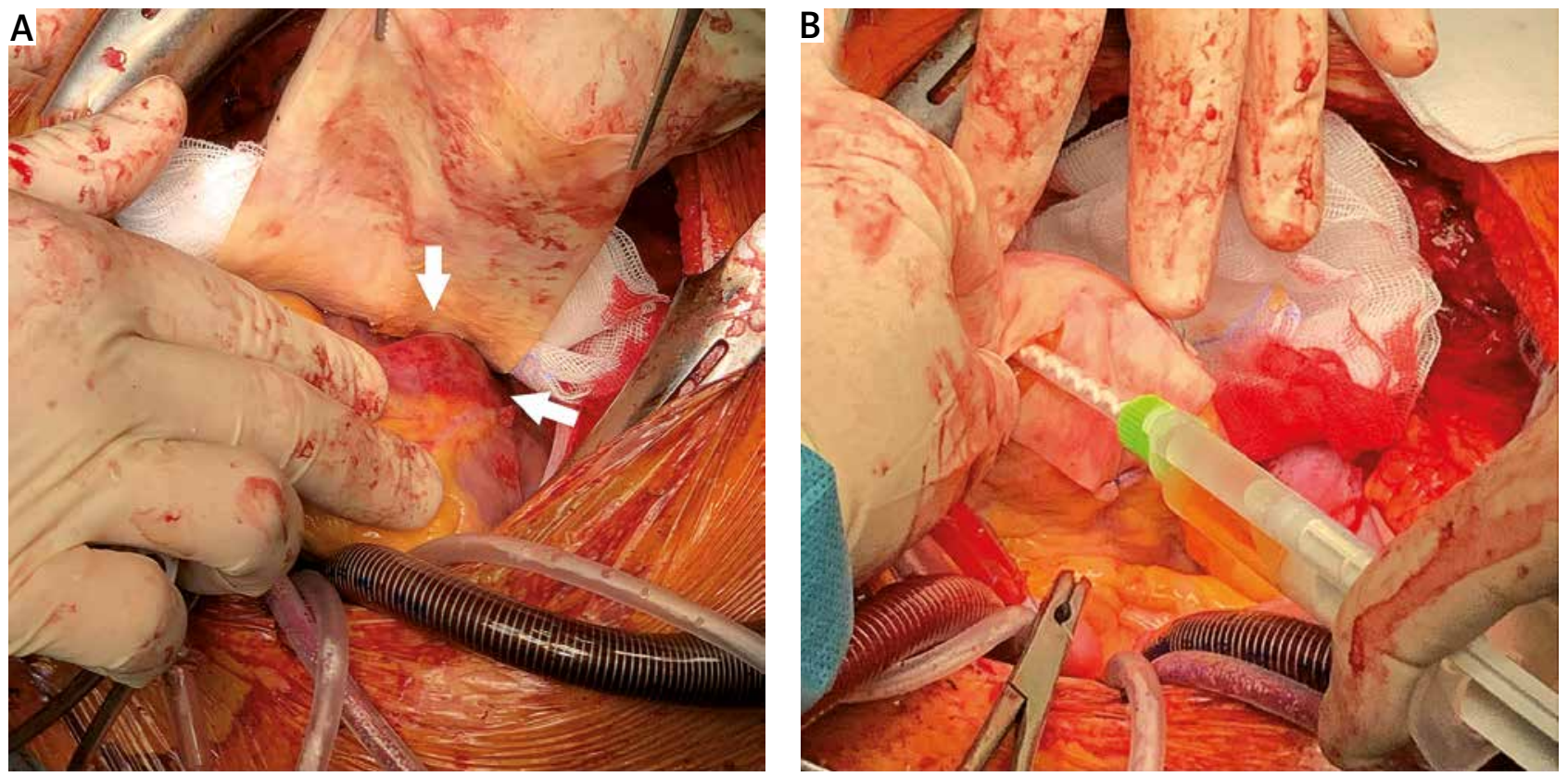

Figure 2. A - Intraoperative view of the area of incomplete left ventricular wall rupture covered by a thin layer of myocardium (arrows). B - Pericardial patch (Bio Integral $8 \times 12 \mathrm{~cm}$ ) fixed onto the lesion with a healthy myocardium margin using surgical glue (BioGlue, CryoLife)

FWR is one of the most serious complications of acute myocardial infarction, frequently leading to death due to severe intra-pericardial bleeding and subsequent cardiac tamponade [1]. The major risk factors for FWR include advanced age, female gender, hypertension, delayed or no reperfusion, and first myocardial infarction [2].

Morphologically, four patterns of FWR have been described. Type I exhibits an almost direct trajectory with small dissection and bloody infiltration of the myocardium. Type II has a multicanalicular trajectory with extensive myocardial dissection and bloody infiltration. In type III, the orifice of the rupture is protected either by thrombus or pericardial symphysis. Type IV rupture is incomplete, with the trajectory not extending through all myocardial layers [3]. The majority of survivors had a rupture of type III or IV [4]. These two categories are usually grouped together under the broad term "pseudoaneurysm" [3-5]. However, some authors suggest that a rupture roofed by visceral pericardium should be described as a "contained myocardial rupture", which is an intermediate stage between complete and incomplete FWR [4].

Echocardiography represents a first-line diagnostic tool for FWR. This approach is characterized by high specificity (90\%) but only moderate sensitivity (70\%) [6]. Therefore in problematic cases, especially in the absence of pericardial effusion, more advanced imaging techniques - cardiac computed tomography and CMR, both providing higher diagnostic accuracy in the identification and determination of FWR extent - should be considered.

Conservative treatment strategies in FWR have been described in the literature, however outcomes of such management make surgical interventions usually the treatment of choice. Sutureless repair techniques, such as that used in the present case, are gaining importance. They are relatively simple, fast, preserve LV geometry and provide complete hemostasis, leaving the necrotic tissues untouched. However, it should be stressed that their use, supported by satisfactory clinical results, is limited to FWR without active pericardial bleeding (type III and IV) [7].

In conclusion, this clinical case demonstrates the usefulness of urgent CMR in early diagnosis of incomplete FWR in the acute phase of myocardial infarction. This imaging approach allowed for planning immediate surgery using a sutureless technique before the rupture of the myocardial wall became complete.

\section{Disclosure}

Authors report no conflict of interest.

\section{References}

1. Reddy SG, Roberts WC. Frequency of rupture of the left ventricular free wall or ventricular septum among necropsy cases of fatal acute myocardial infarction since introduction of coronary care units. Am J Cardiol 1989; 63: 906-911.

2. Verhaegh AJ, Bouma W, Damman K, et al. Successful emergent repair of a subacute left ventricular free wall rupture after acute inferoposterolateral myocardial infarction. J Cardiothorac Surg 2018; 13: 82.

3. Perdigao C, Andrade A, Ribeiro C. Cardiac rupture in acute myocardial infarction. Various clinico-anatomical types in 42 recent cases observed over a period of 30 months. Arch Mal Coeur Vaiss 1987; 80: 336-344.

4. Helmy TA, Nicholson WJ, Lick S, Uretsky BF. Contained myocardial rupture: a variant linking complete and incomplete rupture. Heart 2005; 91: e13.

5. Sutherland F, Guell F, Pathi V, Naik S. Postinfarction ventricular free wall rupture: strategies for diagnosis and treatment. Ann Thorac Surg 1996; 61: 1281-1285.

6. Krishnan U, Mccann GP, Hickey M, Schmitt M. Role of contrast-enhanced magnetic resonance imaging in detecting early adverse remodeling and subacute ventricular wall rupture complicating myocardial infarction. Heart Vessels 2008; 23: 430-432.

7. Matteucci M, Fina D, Jiritano F, et al. Treatment strategies for post-infarction left ventricular free-wall rupture. Eur Hear J Acute Cardiovasc Care 2019; 8: 379-387. 